\title{
Decisions, decisions
}

Standard decision theory postulates that ambiguity about probabilities of winning should not affect choices. However, experiments show that many people are more willing to bet on risky outcomes than on ambiguous ones. This empirical aversion to ambiguity prompted Ming Hsu and colleagues to search for neural distinctions between risk and ambiguity using functional MRI (fMRI).

Decision-making under risky and ambiguous uncertainty is best illustrated by what is known as the Ellsberg paradox. Imagine one deck of 20 cards composed of 10 red and 10 blue cards (the risky deck), and another of 20 red and blue cards in which the composition of red and blue cards is unknown (the ambiguous deck). A bet on a colour pays a fixed sum if a card of the chosen colour is drawn. Which deck of cards would participants prefer to bet on? Interestingly, most people prefer to

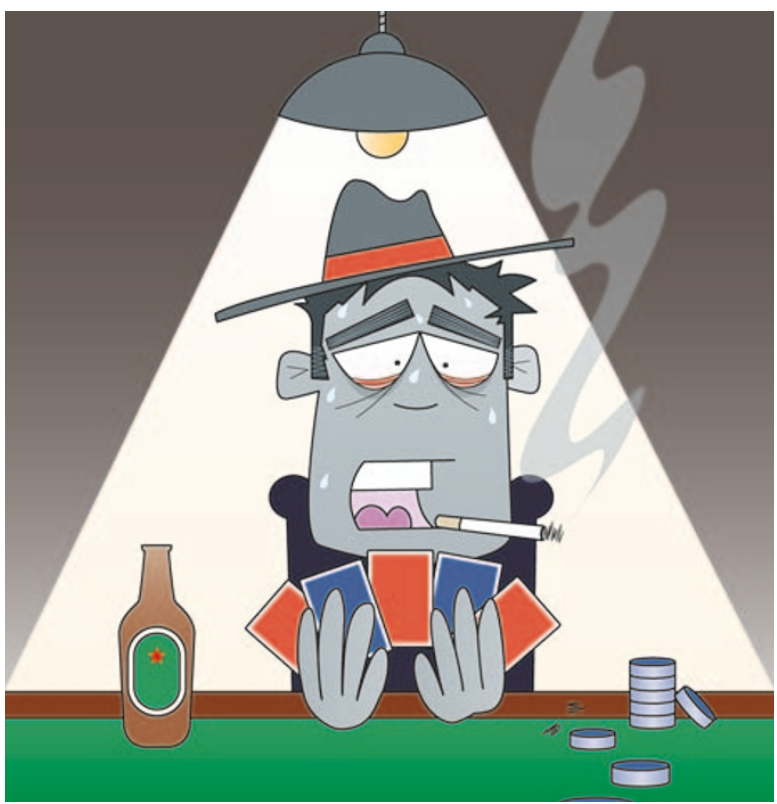

bet on a card from the risky rather than the ambiguous deck, even though there is a $50-50$ chance of winning in both cases.

Using similar experimental treatments, the researchers show that the brain in fact treats the two decks of cards differently. Twenty-four areas in the brain are more active under conditions of ambiguity than risk. Among these regions are the amygdala, the orbitofrontal cortex (OFC) and the dorsomedial prefrontal cortex. By constrast, the dorsal striatum is preferentially activated during the risky condition. As the dorsal striatum is implicated in reward prediction, the result indicates that ambiguity lowers the anticipated reward of decisions.

To confirm the fMRI results, Hsu et al. conducted similar behavioural tests using patients with focal brain lesions. They found that patients with OFC lesions did not distinguish between risky and ambiguous uncer- tainty - an observation that was not seen in control patients who had temporal lobe damage.

Therefore, different areas of the brain are activated when we evaluate ambiguous and risky choices. This might underlie a general process of how organisms explore their environment, which often contains risky and ambiguous uncertainty. In addition, understanding the neural basis of choice under uncertainty has important social implications because it is a fundamental activity at every level of society - from the personal to the political.

Jane Qiu

ORIGINAL RESEARCH PAPER Hsu, M. et al. Neural systems responding to degrees of uncertainty in human decision-making. Science 310, 1680-1683 (2005)

FURTHER READING Sugrue, L. P. et al. Choosing the greater of two goods: neural currencies for valuation and decision making. Nature Rev. Neurosci. 6, 363-375 (2005). WEB SITE

Camerer's laboratory: http://www.hss.caltech. edu/ camerer/camerer.html 